\title{
The Analysis of Electronics Industry Warehouse Process Enhancement
}

\author{
Lienying Chan ${ }^{*}$ and Minglang Wang \\ No.707, Sec.2, Wu-Fu Rd., Hsinchu City, Chung-Hua University, Taiwan \\ ${ }^{*}$ Corresponding Author \\ 高科技产业库房仓储流程分析与改善 \\ 詹莲英*，王明郎 \\ 中华大学, 新竹市, 台湾 \\ 通讯作者
}

\begin{abstract}
This paper is about the analysis of electronics industry warehouse process; it tries to find the significant part need to be enhanced. By utilizing Analytic Hierarchy Process, AHP, we divide the warehouse process into 3 aspects: Stock, Storage and Shipment. We consulted quite a few experts of warehouse and collected their opinion by survey. According to the analytic data of the survey, we find that experts highly pay attention to the criteria: fire-proof \& thief-proof risk control whose weighting is 0.116 and the experts are most indifferent to independent import area whose weighting is 0.024 . So we can make a conclusion as follows, if we want to enhance the electronics industry warehouse process, the first thing we need to do is to control the fire-proof $\&$ thief-proof risk, and the second thing is to assure the amount of shipment. By referring to the analytic result of this paper, we can know the best way to enhance the electronics industry warehouse process.
\end{abstract}

Keywords-warehouse process; analytic hierarchy process; pairwise comparison matrix

摘要一本研究针对高科技产业库房仓储流程分析最关键需 要改善的部分。利用层级分析法 (Analytic Hierarchy Process, AHP) 将整个流程分为进货、仓储以及出货 3 个构面, 访谈专家 并藉由问卷搜集资料。经过统计分析后的结果得到, 各准则中专 家最为重视的是防火防盗风险管控(0.116), 最不重视的是独立进 货暂存区(0.024)。因此可得知如果要针对高科技产业库房仓储流 程做改善, 首先最必须要注意的是防火防盗风险管控, 其次是出 货数量正确性的确保。利用此研究结果可以做为仓储流程改善的 方向与依据。

关键词一仓储流程; 层级分析法; 成对比较矩阵

\author{
I. 前言
}

随着科技不断进步, 高科技电子产业的蓬勃发展是有目 共睹的。为了能在激烈的竞争中脱颖而出, 除了产品创新、 市场开发外, 能否高效管理仓储系统流程也将是一个很重 要的课题。因此本研究针对高科技产业库房仓储流程做分 析, 尝试找出最为重要的项目, 做为相关仓储作业改善的 方向与依据。

\section{II. 层级分析法}

利用 1971 年 Thomas L. Saaty 所发展出的层级分析法 (Analytic Hierarchy Process, AHP)[3], 可以解决在不确定情 况下及具有多数个评估准则的决策问题。它可以轻易地将 复杂的问题系统透过多层级去拆解, 再由各方面去评估比 较, 透过量化的统计并分析, 即可找出问题的关键, 提供 给决策者做为参考, 减少错误决策的风险, 做出最有利的 决策。

层级分析法主要可分为以下步骤: (1)层级的建立是整个 层级分析法中相当重要的部分, 会先将终极目标分成数个 构面, 每个构面再分别细分为数个准则, 由此建立起整个 层级架构。(2)接着再由专家根据专业判断填表建立成对比 较矩阵。(3)最后计算出各层级的优势向量(权重 $W$ ), 并求 得总体优势向量(权重 $W_{T}$ ), 得到优化的结果, 若整体一致 性检查没有问题, 就可以有信心的利用该结果来做出最终 决策。建立成对比较矩阵时, 将评估尺度分为了同样重要、 稍微重要、很重要、非常重要、极为重要这 5 个尺度, 并 分别赋予 $1 、 3 、 5 、 7 、 9$ 的量化数值, 另外为了语意的连 续性, 在每两个基本尺度间各加入一个尺度并赋予 2、4、6、 8 的量化数值。且项目两两比较时, 将采取比例尺度的方式 来完成, 如下表 1 所示。 
表 I 项目比较时的比例尺度

\begin{tabular}{|c|c|c|c|c|c|c|c|c|c|c|c|c|c|c|c|c|c|c|}
\hline & $\begin{array}{c}\text { 极 } \\
\text { 为 } \\
\text { 重 } \\
\text { 要 }\end{array}$ & $\begin{array}{c}\text { 非 } \\
\text { 常 } \\
\text { 重 } \\
\text { 要 }\end{array}$ & $\begin{array}{c}\text { 很 } \\
\text { 重 } \\
\text { 要 }\end{array}$ & $\begin{array}{c}\text { 稍 } \\
\text { 微 } \\
\text { 重 } \\
\text { 要 }\end{array}$ & $\begin{array}{c}\text { 茼 } \\
\text { 样 } \\
\text { 重 } \\
\text { 要 }\end{array}$ & & $\begin{array}{c}\text { 稍 } \\
\text { 微 } \\
\text { 重 } \\
\text { 要 }\end{array}$ & & $\begin{array}{c}\text { 很 } \\
\text { 重 } \\
\text { 要 }\end{array}$ & & $\begin{array}{c}\text { 非 } \\
\text { 常 } \\
\text { 重 } \\
\text { 要 }\end{array}$ & $\begin{array}{c}\text { 极 } \\
\text { 为 } \\
\text { 重 } \\
\text { 要 }\end{array}$ \\
\hline & $9: 1$ & $8: 1$ & $7: 1$ & $6: 1$ & $5: 1$ & $4: 1$ & $3: 1$ & $2: 1$ & $1: 1$ & $1: 2$ & $1: 3$ & $1: 4$ & $1: 5$ & $1: 6$ & $1: 7$ & $1: 8$ & $1: 9$ & \\
\hline $\mathrm{A}$ & & & & & & & & & & & & & & & & & & B \\
\hline
\end{tabular}

若有 $\mathrm{n}$ 个项目就要进行 $\mathrm{n}(\mathrm{n}-1)$ 次比较, 比较结果数值为 $9 、 8 、 \ldots 、 2 、 1 、 1 / 2 、 \ldots, 1 / 8 、 1 / 9$, 将 $\mathrm{n}$ 个项目的比较结 果数值填入成对比较矩阵 $\boldsymbol{A}$ 的上三角形部分, 且主对角线 自身比较的部分都填入 1 , 下三角形部分则填入上三角形部 分数值的倒数。即可建立出成对比较矩阵 $\boldsymbol{A}$, 如下所示:

$$
A=\left[a_{q \eta}\right]=\left[\begin{array}{cccc}
a_{11} & a_{12} & \cdots & a_{1 n} \\
a_{11} & a_{22} & & a_{2 n} \\
\vdots & & \vdots & \vdots \\
a_{n 1} & a_{n 2} & \cdots & a_{n n}
\end{array}\right], a_{q 4}=\frac{1}{a_{j n}}, 1,1=1,2,3, \ldots, n
$$

接着利用列向量几何平均标准化的方式即可求得优势 向量(权重 $W$ ), 方法如下:

$$
W=\left[\begin{array}{c}
W_{1} \\
W_{2} \\
\vdots \\
W_{n}
\end{array}\right], W_{l}=\sqrt[n]{a_{11} \times \sigma_{n 2} \times \ldots \times \sigma_{n 2}}
$$

在符合一致性的情况下 $\left(a_{l j} \times a_{i k}=a_{l k}\right)$, 可求得最大特 征值 $\lambda_{\max }$ 为

$$
=\lambda_{\text {wax }}=\frac{1}{n}\left(A \times W^{\prime}\right)
$$

另外要检查成对比较矩阵是否为一致性矩阵, 就要确认 一致性指标 C.I.值

$$
C_{1} I_{1}=\frac{\lambda_{\text {nลax }}-n}{n-1}
$$
被接受

且只有当一致性比率 C.R.值 $\leqq 0.1$ 时, 矩阵一致性才可

$$
C_{1} R_{1}=\frac{C_{1} I_{1}}{R_{n} I_{1}}
$$

其中 R.I.为随机性指标, 在不同阶数下有不同的数值, 如下表 2 所示
表 II 不同阶数下的随机性指标

\begin{tabular}{|c|c|c|c|c|c|c|c|c|c|c|}
\hline 阶数 & 1 & 2 & 3 & 4 & 5 & 6 & 7 & 8 & 9 & 10 \\
\hline RI & 0 & 0 & 0.58 & 0.9 & 1.12 & 1.24 & 1.32 & 1.41 & 1.45 & 1.49 \\
\hline
\end{tabular}

最后将各构面下的准则优势向量(权重)乘上对应构面的 优势向量(权重), 即可得到加权后各准则的总体优先向量 (总体权重)。将总体权重经过排序后即可得知哪个项目是最 重要的, 最值得去改善的, 将这结果做为决策的参考与依 据。

\section{III. 实验结果}

本研究将高科技产业库房仓储流程分为进货构面、仓储 构面、出货构面等 3 个构面, 各构面再细分如下:

a. 进货：独立进货暂存区、数量点收过程、统一窗口 专责负责人、存货空间充足与否确认、正确货架分类、进 货数据输入供日后追踪等 6 个准则

b. 仓储：妥善的空间安排、分门别类存放、温控湿控 及通风储存环境、防火防盗风险管控、存货数量管控系统 等 5 个准则

c. 出货：快速拣货集货分货、适当的包装避免损伤、 出货数量正确性、出货外箱标示、出货的趟次安排、独立 于进货的出货动线等 6 个准则

透过与专家访谈与问卷发放, 共发放 30 份问卷, 回收 28 份, 问卷回收率为 93\%。经过初步筛选后(剔除有漏答与 规律性填答问卷), 有效问卷数为 23 份, 有效问卷回收率为 $77 \%$ 。

计算构面层级的优先向量(权重)得到以下结果

表 III 构面层级的优先向量(权重)

\begin{tabular}{|c|c|c|}
\hline 排序 & 构面 & W \\
\hline 3 & a. 进货 & 0.311 \\
\hline 1 & b. 仓储 & 0.363 \\
\hline 2 & c. 出货 & 0.325 \\
\hline \multicolumn{2}{|c|}{$\lambda \max =3.001$ C.I. $=0.001$ C.R. $=0.001$}
\end{tabular}

由表 3 可看出专家最为重视的是 b. 仓储构面(0.363), 最 不重视的是 a. 进货构面(0.311), 唯各构面权重差异不大, 代表专家认为各构面皆有一定的重要性, 需要各部份互相 配合才能达到最佳的仓储效益。 


\section{A. 进货构面下各准则的优先向量(权重)}

表 IV 进货构面准则的优先向量(权重)

\begin{tabular}{|c|c|c|}
\hline 排序 & 进货 & W \\
\hline 6 & 独立进货暂存区 & 0.077 \\
\hline 3 & 数量点收过程 & 0.190 \\
\hline 5 & 统一窗口专责负责人 & 0.078 \\
\hline 4 & 存货空间充足与否确认 & 0.128 \\
\hline 2 & 正确货架分类 & 0.228 \\
\hline 1 & 进货数据输入供日后追踪 & 0.299 \\
\hline \multicolumn{2}{|c|}{$\lambda \max =6.019$ C.I. $=0.004$ C.R. $=0.003$}
\end{tabular}

由表 4 可看出进货构面下各准则中专家最为重视的是进 货数据输入供日后追踪(0.299), 原因在于如果能于源头的 地方做好管控登录, 就可以避免货物品项间混杂不清, 造 成后许作业上的困扰; 最不重视的是独立进货暂存区 (0.077), 这是因为该仓储所储存的是高科技电子产品, 产 品品项较为单纯, 因此不被列为急需改善的项目。

\section{B. 仓储构面下各准则的优先向量(权重)}

表 V 仓储构面下各准则的优先向量(权重)

\begin{tabular}{|c|c|c|}
\hline 排序 & 仓储 & W \\
\hline 5 & 妥善的空间安排 & 0.080 \\
\hline 4 & 分门别类存放 & 0.142 \\
\hline 2 & 温控湿控及通风储存环境 & 0.237 \\
\hline 1 & 防火防盗风险管控 & 0.318 \\
\hline 3 & 存货数量管控系统 & 0.224 \\
\hline
\end{tabular}

$\lambda \max =5.168$ C.I. $=0.042$ C.R. $=0.038$

由表 5 可看出仓储构面下各准则中专家最为重视的是防 火防盗风险管控(0.318), 原因在于整个高科技产业中最重 要的在于货物的安全性, 一旦有什么闪失将导致货物有所 减损, 严重将导致整个仓储都付之一炬; 最不重视的是妥 善的空间安排(0.080), 这是因为该仓储所储存的是高科技 电子产品, 各品项的空间安排早已有相当程度的规范, 因 此不被列为急需改善的项目。

\section{C. 出货构面下各准则的优先向量(权重)}

表 VI 出货构面下各准则的优先向量(权重)

\begin{tabular}{|c|c|c|}
\hline 排序 & 出货 & W \\
\hline 6 & 快速拣货集货分货 & 0.084 \\
\hline 2 & 适当的包装避免损伤 & 0.203 \\
\hline 1 & 出货数量正确性 & 0.332 \\
\hline 3 & 出货外箱标示 & 0.196 \\
\hline 5 & 出货的趟次安排 & 0.091 \\
\hline 4 & 独立于进货的出货动线 & 0.094 \\
\hline \multicolumn{2}{|c|}{$\lambda$ max = 6.181 C.I. $=0.036$ C.R. $=0.029$} \\
\end{tabular}

由表 6 可看出出货构面下各准则中专家最为重视的是出 货数量正确性(0.332), 原因在于出货时最需要关注出货的 品项、数量是否符合客户的需求, 若不符合需求将导致整 个出货流程必须重新再跑一遍, 造成作业上旷日费时, 也 会在信誉上产生严重影响; 最不重视的是快速拣货集货分 货 $(0.084)$, 是因为该公司为高科技电子产业, 产品品项较 为单纯, 较不需要复杂的拣货、集货、与分货, 在这些项 目的速度上已经有一定的水平, 故不被列为迫切需要改善 的项目。

\section{D. 加权后各准则的总体优先向量(总体权重)}

表 VII 加权后各准则的总体优先向量(总体权重)

\begin{tabular}{|c|c|c|}
\hline 排序 & 准则 & $\mathrm{W}$ \\
\hline 17 & 独立进货暂存区 & 0.024 \\
\hline 9 & 数量点收过程 & 0.059 \\
\hline 16 & 统一窗口专责负责人 & 0.024 \\
\hline 11 & 存货空间充足与否确认 & 0.040 \\
\hline 6 & 正确货架分类 & 0.071 \\
\hline 3 & 进货数据输入供日后追踪 & 0.093 \\
\hline 14 & 妥善的空间安排 & 0.029 \\
\hline 10 & 分门别类存放 & 0.051 \\
\hline 4 & 温控湿控及通风储存环境 & 0.086 \\
\hline 1 & 防火防盜风险管控 & 0.116 \\
\hline 5 & 存货数量管控系统 & 0.081 \\
\hline 15 & 快速拣货集货分货 & 0.027 \\
\hline 7 & 适当的包装避免损伤 & 0.066 \\
\hline 2 & 出货数量正确性 & 0.108 \\
\hline 8 & 出货外箱标示 & 0.064 \\
\hline 13 & 出货的趟次安排 & 0.030 \\
\hline 12 & 独立于进货的出货动线 & 0.030 \\
\hline
\end{tabular}

由表 7 可看出在各准则中专家最为重视的是防火防盗风 险管控(0.116), 最不重视的是独立进货暂存区(0.024)。

\section{IV. 结论与建议}

本研究针对高科技产业库房仓储流程做分析, 尝试找出 最为重要的项目, 做为相关仓储作业改善的方向与依据。 本研究之研究方法利用层级分析法(Analytic Hierarchy Process, AHP), 经过统计分析后的结果可得知, 如果要针 对高科技产业库房仓储流程做改善, 首先最必须要注意的 是防火防盗风险管控, 其次是出货数量正确性的确保。利 用此研究结果可以做为仓储流程改善的方向与依据, 未来 可进一步地研究采用各种方法(如:装设警示系统)对于改善 仓储流程的成效做评估, 可以给决策者更完善的参考。 


\section{REFERENCES}

[1] C. Y. Deng and K. H. Tseng(1989), The essential properties and applications of Analytic Hierarchy Process - part 1, China Statistics, Vol. 27, No. 6, pp. 13707-13724.

[2] C. Y. Deng and K. H. Tseng(1989), The essential properties and applications of Analytic Hierarchy Process - part 2, China Statistics, Vol. 27, No. 7, pp. 13767-13870.

[3] Saaty, T. L., (1971), How to make a decision: The analytic hierarchy process. European Journal of Operational Research, 40, pp. 9-10.

[4] Saaty, Thomas L., (1977), “ A Scaling Method for Priorities in Hierarchical Structure, " Journal of Mathematical Psychology, Vol. 15, No. 3, pp. 234-281.

[5] Saaty, T. L., (1980), “The Analytic Hierarchy Process”, McGraw-Hill, New York. 